\title{
Adjuvant role of macrophages in stem cell-induced cardiac repair in rats
}

\author{
Soo yeon Lim', Dong Im Cho', Hye-yun Jeong ${ }^{1}$, Hye-jin Kang ${ }^{1}$, Mi Ra Kim¹, Meeyoung Cho', Yong Sook Kim ${ }^{1,2}$ and \\ Youngkeun Ahn ${ }^{1,3}$
}

\begin{abstract}
Bone marrow-derived mesenchymal stem cells (BMMSCs) are used extensively for cardiac repair and interact with immune cells in the damaged heart. Macrophages are known to be modulated by stem cells, and we hypothesized that priming macrophages with BMMSCs would enhance their therapeutic efficacy. Rat bone marrow-derived macrophages (BMDMs) were stimulated by lipopolysaccharide (LPS) with or without coculture with rat BMCs. In the LPS-stimulated BMDMs, induction of the inflammatory marker iNOS was attenuated, and the anti-inflammatory marker Arg1 was markedly upregulated by coculture with BMMSCs. Myocardial infarction (MI) was induced in rats. One group was injected with BMMSCs, and a second group was injected with MIX (a mixture of BMMSCs and BMDMs after coculture). The reduction in cardiac fibrosis was greater in the MIX group than in the BMC group. Cardiac function was improved in the BMMSC group and was substantially improved in the MIX group. Angiogenesis was better in the MIX group, and anti-inflammatory macrophages were more abundant in the MIX group than in the BMMSC group. In the BMMSCs, interferon regulatory factor 5 (IRF5) was exclusively induced by coculture with macrophages. IRF5 knockdown in BMMSCs failed to suppress inflammatory marker induction in the macrophages. In this study, we demonstrated the successful application of BMDMs primed with BMMSCs as an adjuvant to cell therapy for cardiac repair.
\end{abstract}

\section{Introduction}

To date, inducing cardiac regeneration using stem cells has remained tremendously challenging. Bone marrowderived mesenchymal stem cells (BMMSCs) are regarded as an attractive option for cardiac regeneration therapy ${ }^{1}$. BMMSCs are relatively easy to isolate and can differentiate into mesenchymal lineage cell types, such as osteocytes, chondrocytes, adipocytes, and myocytes ${ }^{2}$. Additionally, MSCs have the immunoprivileged capacity to avoid rejection, suppress inflammation in the lesion, and modulate immune cell phenotypes ${ }^{3}$. Despite the safety and feasibility of BMMSCs, concerns over their use

\footnotetext{
Correspondence: Yong Sook Kim (retissue@jnu.ac.kr) or

Youngkeun Ahn (cecilyk@hanmail.net)

${ }^{1}$ Cell Regeneration Research Center, Chonnam National University Hospital,

Gwangju 61469, Republic of Korea

2Biomedical Research Institute, Chonnam National University Hospital,

Gwangju 61469, Republic of Korea

Full list of author information is available at the end of the article.

These authors contributed equally: Yong Sook Kim, Youngkeun Ahn
}

remain due to equivocal clinical outcomes ${ }^{4}$. Various modifications have been proposed to improve the therapeutic efficacy of BMMSCs in cardiovascular disorders, such as priming with growth factors, cardiogenic cocktails, or apicidin or hypoxia-enhanced functional restoration via angiogenesis and cardiac differentiation ${ }^{5-7}$.

Macrophages are highly dynamic immune cells with diverse functions and are widely involved in the pathogenesis of cardiovascular diseases, including myocardial infarction (MI) and atherosclerosis. After MI, different leukocyte populations dynamically infiltrate the heart ${ }^{8}$. Importantly, anti-inflammatory macrophages are essential for inducing infarct healing, because depletion of cardiac macrophages drastically impairs healing and worsens the disease outcome9. Moreover, infarct healing and repair by BMMSCs is mediated by macrophages ${ }^{10}$. We previously reported that macrophages were shifted toward the anti-inflammatory phenotype by coculture with BMMSCs ${ }^{11}$. 
Given the important contribution of anti-inflammatory macrophages to infarct healing, we hypothesized that macrophages cocultured with BMMSCs would be safe and effective adjuvant cells for cell therapy. To prove this concept, we prepared a mixture of BMMSCs and antiinflammatory macrophages isolated and differentiated from bone marrow (BMDMs) and investigated their therapeutic efficacy in a rat MI model.

\section{Materials and Methods}

\section{Cytokines and reagent}

Recombinant rat interleukin-4 (IL-4), IL-13, and interferon- $\gamma$ (IFN- $\gamma$ ) were purchased from Life Technologies (Grand Island, NY, USA). Lipopolysaccharide (LPS) was purchased from Sigma-Aldrich (St. Louis, MO, USA).

\section{Cell culture}

Rat BMMSCs (rBMMSCs) were cultured in DMEM with 10\% FBS (Gibco) and 1\% penicillin/streptomycin (Gibco, Grand Island, NY, USA). The cells were grown at $37^{\circ} \mathrm{C}$ in a humidified atmosphere with $95 \%$ air and $5 \% \mathrm{CO}_{2}$, and the medium was changed every 3 days. On reaching confluence, the cells were resuspended with $0.25 \%$ trypsin-EDTA (Gibco) and reseeded at a 1:2 split ratio. The rBMMSCs were used within three passages. Human BMMSCs (hBMMSCs) immortalized by the introduction of telomerase were kindly provided by Professor Yeon-Soo Kim (Inje University, Inje, South Korea). Rat bone marrow cells or THP-1 cells were cultured in RPMI-1640 medium (Gibco) supplemented with macrophage differentiation medium (30\% L929 cell-conditioned medium, 20\% FBS, and 50\% RPMI-1640). L929 cell-conditioned medium was prepared by growing L929 cells in RPMI-1640 medium containing $10 \%$ FBS for 10 days. The medium was harvested and passed through a $0.22-\mu \mathrm{m}$ filter. The macrophages were treated with LPS $(100 \mathrm{ng} / \mathrm{mL}) / \mathrm{IFN}-\gamma(30 \mathrm{ng} / \mathrm{mL})$ or IL-4 $(20 \mathrm{ng} / \mathrm{mL}) / \mathrm{IL}-13(20 \mathrm{ng} / \mathrm{mL})$ to induce polarization.

\section{Coculture of rBMDMs and rBMCs}

For coculture, differentiated rBMDMs were seeded into a six-well plate. The next day, rBMMSCs were placed into 0.4-mm-pore size Corning Transwell culture plate inserts (Sigma-Aldrich).

\section{Real-time PCR}

To compare mRNA expression levels, cells were harvested and homogenized in TRIzol solution (Invitrogen, Carlsbad, CA, USA) according to the manufacturer's instructions. cDNA was generated using the TaqMan MicroRNA Reverse Transcription kit (Applied Biosystems, Foster Ckty, CA, USA), and real-time PCR was performed using the QuantiTect SYBR Green PCR Kit (Qiagen, Hilden, Germany) and Corbett Research RotorGene RG-3000 Real Time PCR System. The sequencespecific human primers were purchased and rat primers were synthesized (Bioneer, Daejeon, Korea). The rat primers are described in Table 1.

\section{Western blotting}

Whole cell lysates were harvested using lysis buffer (20 $\mathrm{mM}$ Tris-HCl pH 7.4, $0.1 \mathrm{mM}$ EDTA, $150 \mathrm{mM} \mathrm{NaCl}, 1$ $\mathrm{mM}$ phenylmethylsulfonyl fluoride, and $1 \mathrm{mg} / \mathrm{mL}$ of leupeptin) on a rotation wheel for $1 \mathrm{~h}$ at $4{ }^{\circ} \mathrm{C}$. After centrifugation at $10,000 \times g$ for $10 \mathrm{~min}$, the supernatant was prepared as a protein extract. Equal concentrations of proteins were fractionated by electrophoresis on 8 to $12 \%$ acrylamide gels and transferred onto a polyvinylidene fluoride membrane (Merck Millipore, Darmstadt, Germany). The membranes were blotted with antibodies against iNOS, Arg1, IRF5 (Cell Signaling Technology, Danvers, MA, USA) and GAPDH (Santa Cruz Biotech, Dallas, TX, USA), followed by secondary antibodies. Protein expression was detected using an Image Reader (LAS-3000 Imaging System, Fuji Photo Film, Tokyo, Japan).

\section{Cell transplantation in a rat myocardial infarction model}

Male inbred SD mice (approximately 7-8 weeks of age) were purchased from SAMTAKO Inc. (Korea). All experiments were performed after receiving approval from our local ethics committee at Chonnam National University Medical School (CNU IACUC-H-2014-22).

Table 1 Primer sequences for the real-time PCR analysis

\begin{tabular}{|c|c|c|}
\hline Gene & Forward primer & Reverse primer \\
\hline Rat iNOS & TCACCTTCGAGGGCAGCCGA & CAGACGCCATGGTGCAGGGG \\
\hline Rat Arg1 & ATTCACCCCGGCTACGGGCA & AGGAGCAGCGTTGGCCTGGT \\
\hline Rat IL-1 $\beta$ & ACCTGTCCTGTGTGATGAAAG & CTCCACTITGGTCTTGACTTCT \\
\hline Rat TNF-a & GCAGATGGGCTGTACCTTATC & GAAATGGCAAATCGGCTGAC \\
\hline Rat GAPDH & GGCCAAGGTCATCCATGA & TCAGTGTAGCCCAGGATG \\
\hline Rat CD206 & GACGGACGAGGAGTTCATTATAC & GTTGGAGAGATAGGCACAGAAG \\
\hline Rat IL-10 & AGTGGAGCAGGTGAAGAATG & GAGTGTCACGTAGGCTTCTA \\
\hline
\end{tabular}


The mice were anesthetized with an intramuscular injection of ketamine $(50 \mathrm{mg} / \mathrm{kg})$ and xylazine $(5 \mathrm{mg} / \mathrm{kg})$, intubated, and mechanically ventilated. The proximal left anterior descending coronary artery was ligated. For transplantation into the hearts, rBMMSCs $\left(5 \times 10^{5}\right.$ diluted in $200 \mu \mathrm{L}$ of PBS) and a mixture of rBMDMs and rBMMSCs $\left(5 \times 10^{5}\right.$ diluted in $200 \mu \mathrm{L}$ of PBS) were prepared. PBS $(n=11)$, rBMCs $(n=8)$, or MIX (rBMDMs + rBMMSCs, $n=8$ ) was injected into the peri-infarct area of the left ventricular myocardium. Finally, the heart was repositioned in the chest, and the chest was closed. The animals remained in a supervised setting until they fully regained consciousness.

\section{Cardiac function measurement}

At 2 weeks, cardiac function was assessed by transthoracic echocardiography $(15-\mathrm{MHz}$ linear array transducer system; iE33 system, Philips Medical Systems; Amsterdam, the Netherlands).

\section{Histological analyses and immunohistochemistry}

For the immunohistochemical analysis, the heart was harvested, embedded in Tissue Tek O.C.T. compound (Leica, Germany), and frozen in liquid nitrogen. Frozen tissues were cut at a thickness of $10 \mu \mathrm{m}$ and mounted onto glass slides for staining. Cardiac fibrosis was measured by Masson's trichrome staining, and fibrotic changes were evaluated by measuring blue-stained fibrotic deposits using an Eclipse-80i microscope and the NISElements software (Nikon, Tokyo, Japan). The percentage of ventricular fibrosis was calculated as the blue-stained area divided by the total ventricular area. For the immunohistochemical analysis, the slides were fixed with acetone for $10 \mathrm{~min}$, permeabilized with $0.1 \%$ Triton X-100 for $10 \mathrm{~min}$, and treated with 3\% hydrogen peroxide in PBS for $10 \mathrm{~min}$ at room temperature to block endogenous peroxidase activity. After blocking nonspecific binding with $5 \%$ normal goat serum or $5 \%$ normal horse serum (Vector Laboratories, Burlingame, CA, USA) at room temperature for $1 \mathrm{~h}$, the excess serum was removed. The slides were incubated with primary antibodies against CD68 (BMA Biomedicals, Switzerland, 1:100), CD206 (Abcam, Cambridge, MA, USA, 1:100), and von Willebrand Factor (vWF; Sigma-Aldrich) for $24 \mathrm{~h}$ at $4{ }^{\circ} \mathrm{C}$. The sections were washed with PBS three times and then incubated for $1 \mathrm{~h}$ with Alexa-Fluor 488 or 594 secondary antibodies. After washing, the slides were coverslipped with mounting medium (VectaMount mounting medium, Vector Laboratories). Images were obtained using the NISElements Advanced Research Program (Nikon, Japan).

\section{siRNA transfection}

RNA interference was performed according to the manufacturer's protocols. Briefly, a siRNA-control (si-
Con) and siRNA specific for IRF5 (si-IRF5) were purchased from Bioneer (Daejeon, South Korea). hBMCs were transfected with the siRNAs using the Lipofectamine RNAiMAX transfection reagent (Invitrogen). The cells were allowed to grow for another $48 \mathrm{~h}$ before being collected for the IRF5 knockdown experiments.

\section{Statistical analysis}

Each experiment was performed at least three times. The data are presented as the means \pm SDs. Differences were analyzed by Student's $t$-test or ANOVA and were considered significant when the $P$-value was $<0.05$.

\section{Results}

rBMMSCs and rBMDMs were isolated and differentiated for coculture. First, the mRNA expression levels of inflammatory mediators were measured in LPSstimulated rBMDMs. Coculture with rBMMSCs reduced the mRNA induction of proinflammatory genes, including iNOS, TNF- $\alpha$, and IL- $1 \beta$, whereas the anti-inflammatory Arg1 mRNA was upregulated in the LPS-stimulated rBMDMs (Fig. 1a). Then, the mRNA levels of antiinflammatory mediators were assessed. Arg1, CD206 and IL-10 were highly induced by culture of the IL-4/IL13-treated rBMDMs with the rBMMSCs (Fig. 1b). Because proinflammatory iNOS and anti-inflammatory Arg1 are the representative markers in rodent monocytes and macrophages, the iNOS to Arg1 ratio was expressed as an indicator of the rBMDM inflammatory status. As shown in Fig. 1c, coculture with rBMMSCs drastically suppressed the inflammatory phenotype of the rBMDMs. Then, the iNOS and Arg1 protein levels were determined by Western blotting. Arg1 was highly increased in LPSstimulated rBMDMs cocultured with rBMMSCs. However, the iNOS levels were not significantly different (Fig. 1d). In the IL-4/IL-13-treated rBMDMs, Arg1 was markedly upregulated, whereas iNOS induction was blocked by BMMSC coculture (Fig. 1e). The overall expression pattern of inflammatory mediators showed high induction of anti-inflammatory Arg1, as well as low expression of proinflammatory mediators in rBMDMs cocultured with rBMMSCs. In addition to iNOS and Arg1, soluble factors released from the rBMDMs were analyzed using an antibody array. The rBMDMs were treated with LPS with or without coculture with rBMMSCs. After $24 \mathrm{~h}$, the culture media were collected for the analysis. In the antibody array, TNF- $\alpha$ was reduced and VEGF, CXCL5, IL-6, and IL-10 were increased in BMDMs cocultured with BMMSCs compared with the levels in BMDMs cultured alone (Fig. 2).

These results showed that the phenotype of the rBMDMs clearly shifted to anti-inflammatory after coculture with rBMMSCs. Thus, we studied whether 


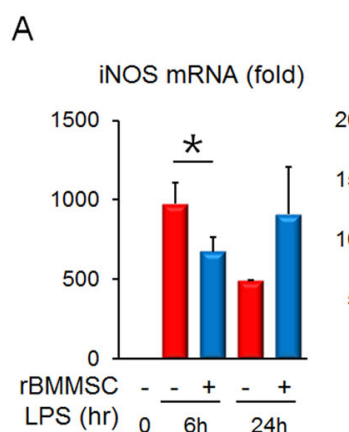

\title{
rBMDM(LPS)
}

\author{
IL-1 $\beta$ mRNA (fold)
}

TNF- $\alpha$ mRNA (fold)

Arg1 mRNA (fold)

LPS (hr) $0 \frac{-1}{6 h} \frac{-1}{24 h}$

B
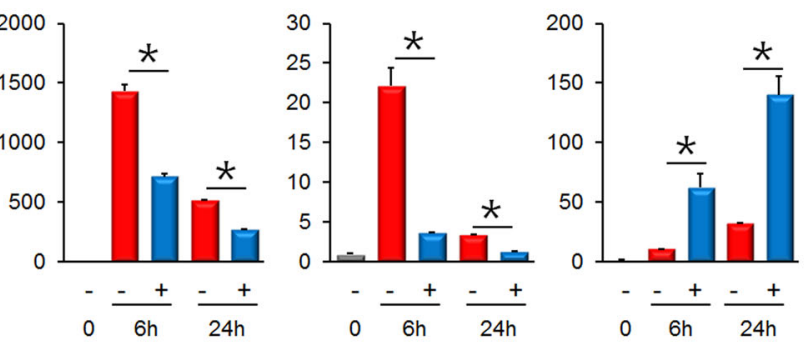

rBMDM(IL4/IL13)

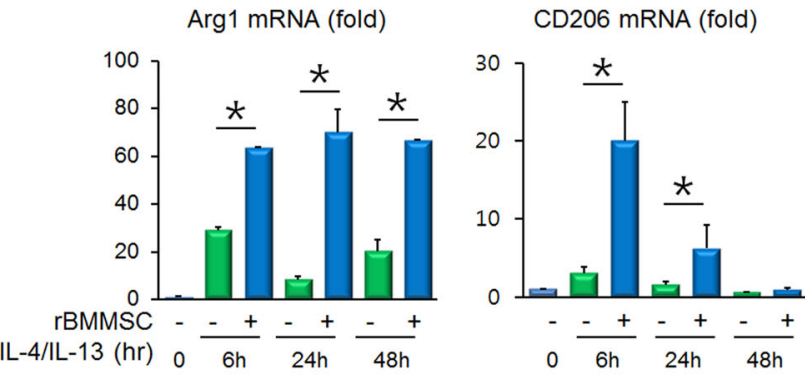

C

iNOS mRNA/Arg1 mRNA (fold)

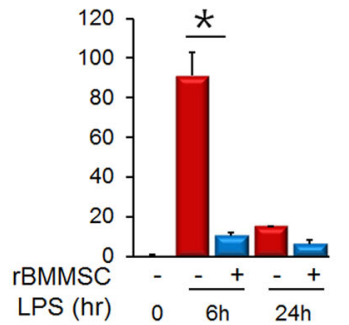

D

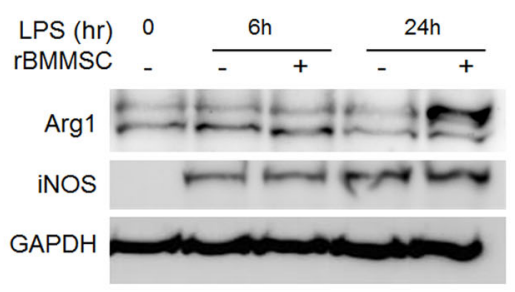

IL-10 mRNA (fold)

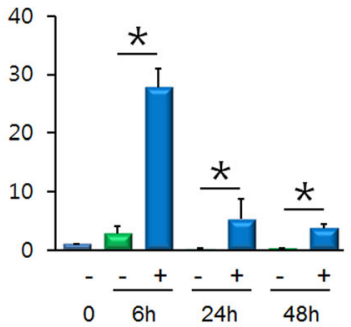

E

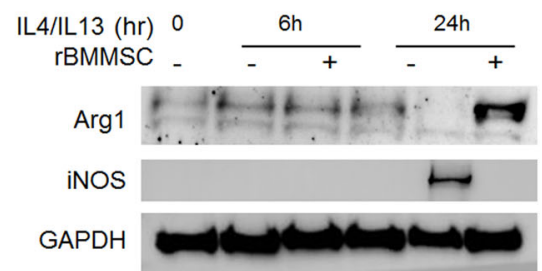

Fig. 1 Expression of inflammation-related mediators in rBMDMs is altered by coculture with rBMMSCs. a The mRNA levels of factors such as iNOS, IL-1 $\beta$, TNF- $a$, and Arg1 were assessed in LPS-stimulated BMDMs with or without rBMMSC coculture. $\mathbf{b}$ The mRNA levels of factors such as Arg 1 , CD206, and IL-10 were assessed in IL-4/IL-13-treated BMDMs with or without rBMMSC coculture. c The iNOS mRNA to Arg1 mRNA ratio was calculated to show the skewed macrophage polarization toward the anti-inflammatory phenotype. The Arg1 and iNOS protein expression patterns in LPS-stimulated rBMDMs (d) or IL-4/L-13-treated rBMDMs (e) with or without rBMMSC coculture. rBMDMs Rat bone marrow-derived macrophages; rBMMSCs Rat bone marrow-derived mesenchymal stem cells

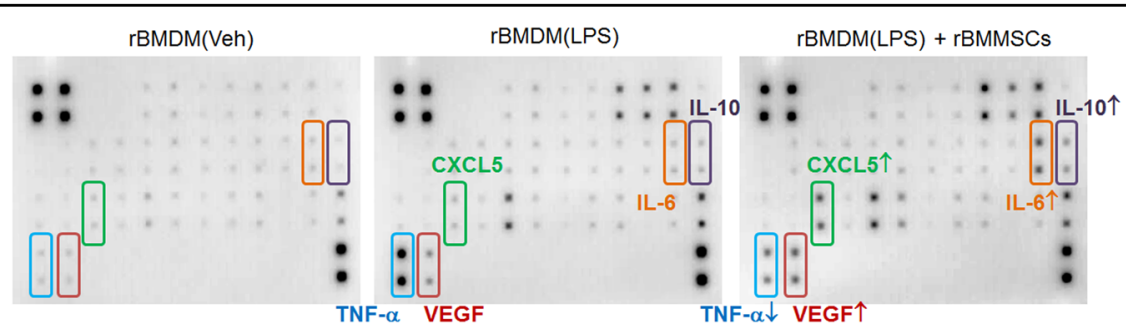

Fig. 2 Antibody array showing that the soluble mediator profiles from BMDMs are altered by coculture with BMMSCs. rBMDM (Veh) Vehicletreated rat BMDMs; rBMDM (LPS) LPS-stimulated rat BMDMs; rBMDM (LPS) + rBMMSCs, LPS stimulation of rBMDMs followed by coculture with rBMMSCs 
the anti-inflammatory rBMDMs would support the therapeutic efficacy of rBMMSCs in a rat MI model. To this end, we injected PBS, rBMMSCs, or a mixture of rBMMSCs and rBMDMs (MIX) into the infarcted myocardium and assessed cardiac fibrosis and function after 2 weeks. The extents of cardiac fibrosis determined by Masson's trichrome staining were $26.1 \pm 3.3 \%, 19.8 \pm$
$2.1 \%$, and $15.9 \pm 1.9 \%$ in the PBS, rBMMSC, and MIX groups, respectively (Fig. 3a). Cardiac function was assessed by echocardiography (Fig. 3b). The MI characterization exhibited better improvement in the MIX group than in the rBMMSC group, although systolic function indexes, such as LVEF, SV and LVFS, also recovered in the rBMMSC group (Fig. 3c).

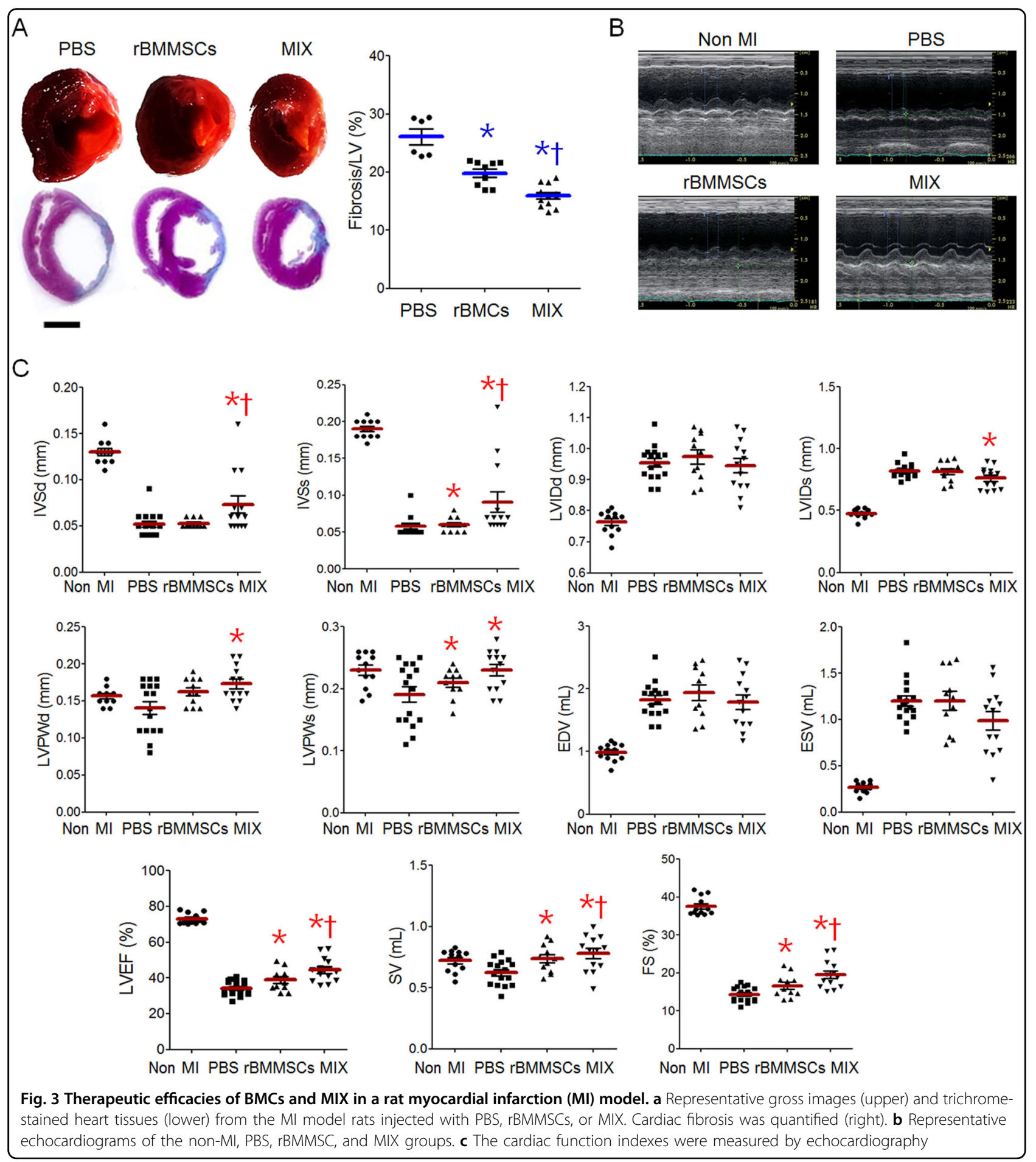


Furthermore, angiogenesis and CD68-positive macrophage infiltration were determined by immunohistochemical staining. The increase in the number of vWFpositive cells was larger in the MIX group than in the rBMMSC group (Fig. 4a). Infiltration of CD68-positive cells was reduced in the rBMMSC group and was much lower in the MIX group than in the PBS group (Fig. 4b).

Next, we determined the phenotype of macrophages in the infarcted heart by double immunohistochemical staining for CD68 (a macrophage marker) and CD206 (an anti-inflammatory macrophage marker). In the confocal images, double-positive cells were more frequently observed in the MIX group than in the rBMMSC group (Fig. 5a). Anti-inflammatory macrophages were quantified by counting CD68-positive and CD206-positive cells; the number of these cells was much higher in the MIX group than in the rBMMSC group (Fig. 5b).

Next, we analyzed the gene expression patterns in human BMCs to define the factor responsible for driving macrophages toward an anti-inflammatory phenotype and selected interferon regulatory factor 5 (IRF5) as a prominent candidate. Hereafter, human BMMSCs (hBMMSCs) and differentiated human THP-1 cells were used due to technical problems, such as transfection difficulty. In the hBMMSCs, the IRF mRNA expression patterns were analyzed. A huge increase in IRF5 was seen in hBMMSCs cocultured with LPS-stimulated macrophages (Fig. 6a). Additionally, IRF5 protein expression was induced by coculture with macrophages with both the hBMMSCs and rBMMSCs (Fig. 6b, c).

Next, to determine whether IRF5 was a critical factor in BMCs for exertion of anti-inflammatory effects on macrophages, IRF5 knockdown was induced in hBMMSCs by siRNA transfection. CXCL10 and CXCL11 were used as inflammatory markers of differentiated THP-1 macrophage cells. The IRF5-knockdown hBMMSCs failed to inhibit the mRNA induction of CXCL10 and CXCL11 (Fig. 6d). Moreover, LPS-induced CXCL10 protein expression was significantly inhibited by coculture with control siRNA-transfected hBMMSCs but was partially restored by coculture with IRF5 siRNAtransfected hBMMSCs (Fig. 6e).

\section{Discussion}

The results of the present study suggest an adjuvant role for anti-inflammatory macrophages as efficient stem cell therapy for MI. Macrophages are key effectors of immune cells in response to tissue injury and are emerging as therapeutic targets for inflammationrelated disorders, including MI and atherosclerosis ${ }^{12-15}$. The murine heart contains myeloid cells with approximately 7 to $8 \%$ noncardiomyocytes ${ }^{16}$, and inflammatory macrophages have been reported to contribute to the initiation and propagation of adverse cardiac remodeling
A

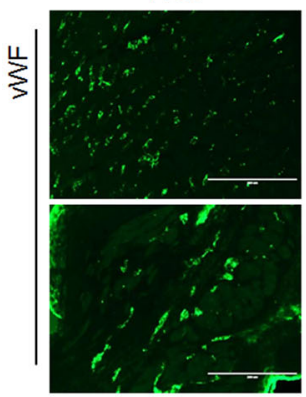

B

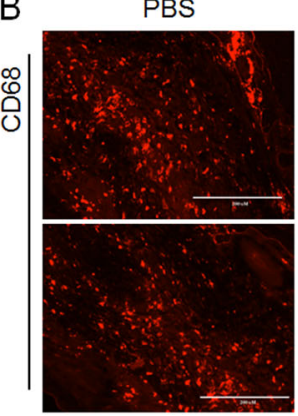

rBMMSCs

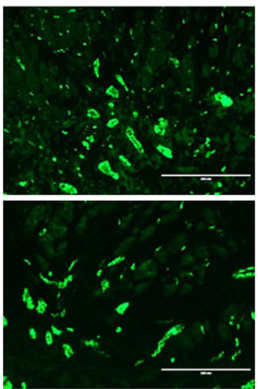

rBMMSCs

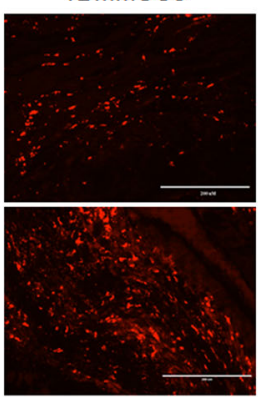

$\mathrm{MIX}$

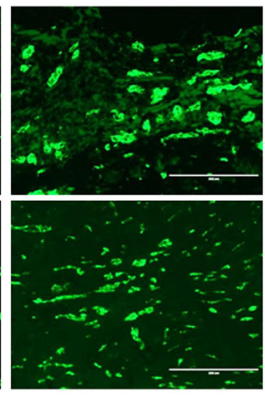

MIX

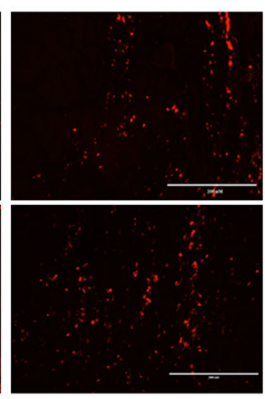

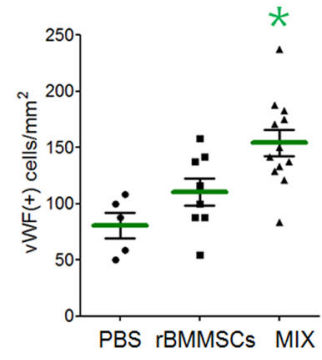

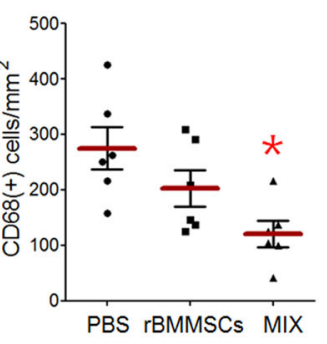

Fig. 4 Angiogenesis and macrophage infiltration in the infarcted myocardium. a The infarcted myocardium was immunostained for vWF to analyze angiogenesis in the PBS, rBMMSC, and MIX groups, and VWF-positive cells were counted for quantification. $\mathbf{b}$ Immunostaining with CD68 showed macrophage infiltration, and the number of CD68-positive cells was quantified 

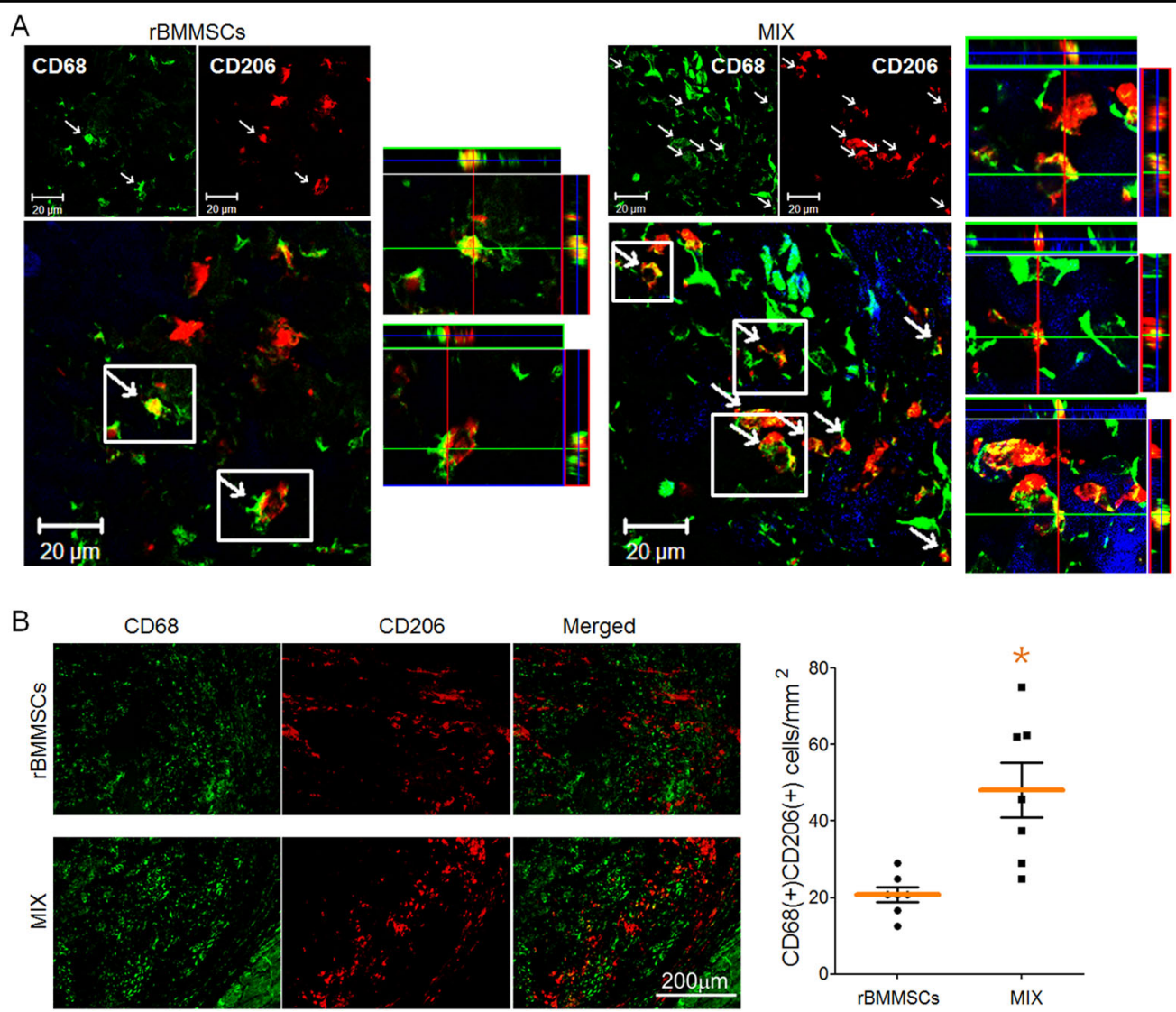

Fig. 5 Representative images showing that anti-inflammatory macrophages are more abundant in the MIX group than in the BMMSC group. a Anti-inflammatory macrophages were detected by double staining with the macrophage marker CD68 and the anti-inflammatory marker CD206 in confocal images. $\mathbf{b}$ The number of anti-inflammatory macrophages identified by double-positive staining for CD68 and CD206 was higher in the MIX group than in the rBMMSC group

during cardiovascular healing processes. The associations between macrophage subsets and functional repair have been validated in independent studies.

The main cells used for stem cell therapy for cardiac regeneration are MSCs, cardiac progenitor cells, and differentiated endothelial cells ${ }^{17-19}$. In clinical studies of ischemic heart disease, improvement was shown in the left ventricular ejection fraction in only 8 of 22 trials, and this analysis concluded that adult stem cell therapy was not ready for routine clinical application ${ }^{20}$. The field of cell therapy for cardiovascular disease has undergone extensive studies and clinical studies using BMMSCs, which exhibit safety profiles and moderate improvement of acute MI and heart failure. However, therapeutic efficacy should be studied in large randomized controlled trials with long-term follow-up. Multiple translational failures led researchers to return to the benches and develop distinguished approaches, such as combined transplantation of different stem cell types. An ongoing clinical study (CONCERT-HF) is evaluating the feasibility, safety, and effect of a combination of
MSCs and c-kit(+) cardiac stem cells on heart failure $(\mathrm{NCT} 02501811)^{21}$.

A great deal of supportive data are available concerning the functions of cardiac macrophages under physiological and pathological conditions. Although the diverse macrophage phenotypes have not been fully characterized, macrophages have been classified into several subsets to clarify their functions. Inflammatory monocytes and macrophages are predominant in the early phase after infarction. These cells produce inflammatory molecules, mediate proteolysis, and phagocytose cell debris in the infarcted heart tissue. In the later stages, alternatively activated macrophages are involved in the repair process and contribute to wound healing and angiogenesis through the production of anti-inflammatory cytokines and growth factors ${ }^{22,23}$.

The induction of anti-inflammatory cytokines is initiated by apoptotic cell ingestion in macrophages. Application of phosphatidylserine-presenting liposomes to mimic apoptotic cells shifted the macrophage phenotype to anti-inflammatory and resulted in the successful 

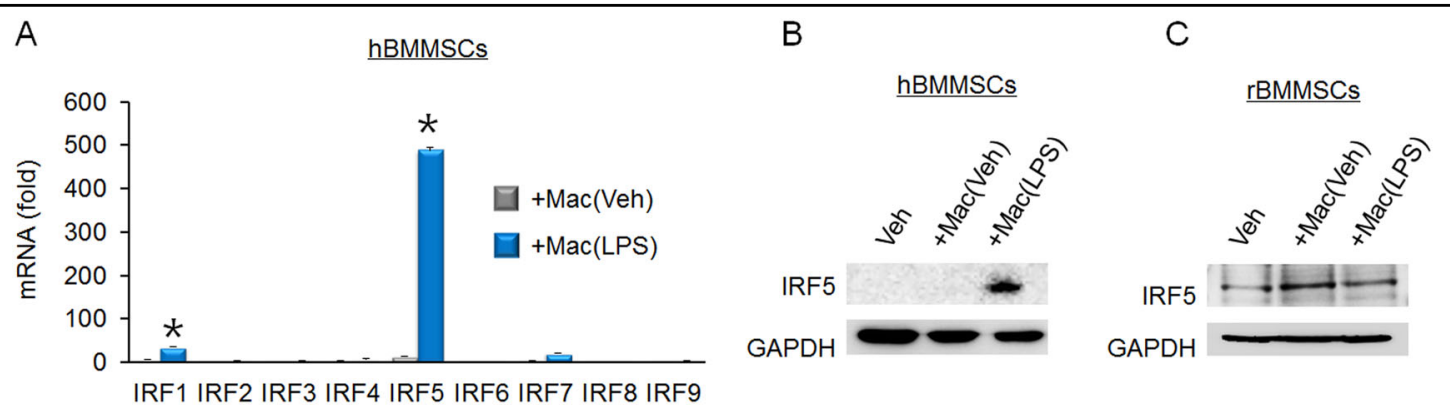
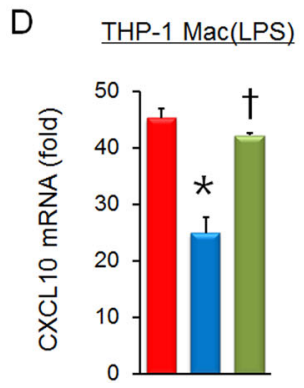

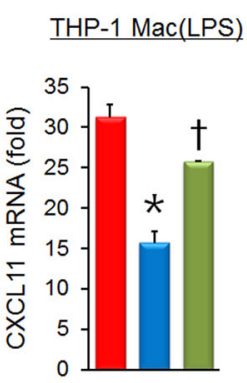

\section{E}
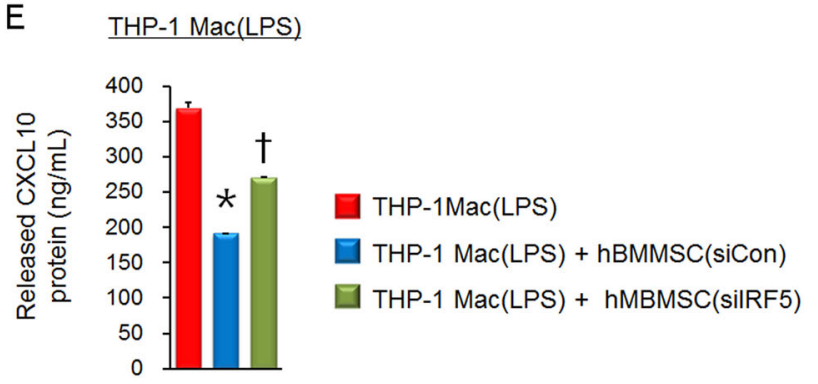

Fig. 6 IRF5 is essential in BMCs and exerts an anti-inflammatory effect on LPS-stimulated macrophages. a mRNA expression patterns of IRF family members were examined in hBMMSCs after coculture with THP-1-derived macrophages stimulated with vehicle or LPS. b IRF5 protein expression was upregulated in hBMMSCs cocultured with LPS-stimulated macrophages. c IRF5 protein expression also increased in rBMMSCs cocultured with rBMDMs. $\mathbf{d}$ LPS-induced upregulation of the proinflammatory markers CXCL10 and CXCL11 was apparently inhibited by coculture with hBMMSCs transfected with the siRNA control. Conversely, coculture with hBMMSCs transfected with the IRF5 siRNA failed to attenuate the upregulation of CXCL10 and CXCL11. e CXCL10 protein expression was measured in media from THP-1-differentiated macrophages cocultured with hBMMSCs transfected with the siRNA control or IRF5 siRNA

prevention of left ventricle remodeling and heart dilatation $^{24,25}$. Interestingly, the depletion of macrophages in mice with MI increased mortality, left ventricular rupture and fibrosis ${ }^{26,27}$. Furthermore, macrophages were required for heart regeneration in stem cell therapy, including MSCs and cardiosphere-derived cells ${ }^{10,28}$, and transplant of these stem cells into the infarcted myocardium induced macrophage polarization toward the reparative phase.

As discussed above, we realized that anti-inflammatory macrophages were clearly involved in the cardiac repair process, and we designed the present study to recapitulate the reparative microenvironment with anti-inflammatory macrophages. Recently, MSCs have been well recognized as a modulator of the immune response that can attenuate the systemic inflammatory response mediated by macrophages $^{11,29}$. Therefore, we harvested anti-inflammatory macrophages after coculture with BMMSCs and then transplanted the mixture of BMMSCs and cocultured macrophages into infarcted heart tissue. This protocol was designed to show that macrophages educated by BMMSCs could be utilized as a cellular adjuvant to limit pathological progression.

A cell therapy product has a concept similar to ours in terms of the cell composition and source. Ixmyelocel-T was developed by culturing a patient's bone marrow in an automated closed-culture system over 11-13 days ${ }^{30}$. Further analysis showed that the ixmyelocel- $T$ product was primarily composed of CD90 $(+)$ mesenchymal stromal cells and CD14(+) macrophages with high expression levels of anti-inflammatory markers, including IL-10, CD163 and CD206 ${ }^{31}$. Moreover, ixmyelocel-T showed an enhanced cholesterol-efflux capacity and was suggested to be beneficial for atherosclerosis ${ }^{32}$. In clinical trials, IMPACT-DCM, ixCELL-DCM, and ixmyelocel-T were applied to treat dilated cardiomyopathy via a catheterbased transendocardial injection ${ }^{33,34}$. A clinically meaningful reduction of cardiac events was observed in patients with ischemic dilated cardiomyopathy but not in those with nonischemic dilated cardiomyopathy. Structural changes in the left ventricular cavity size were not noted in the ixmyelocel-T group at month 12 .

IRF5 is a definite factor for commitment to the inflammatory macrophage lineage, is reported to be expressed on neutrophils ${ }^{35,36}$, and plays an important role in controlling the innate immune response ${ }^{37}$. Silencing IRF5 improves infarct healing and attenuates heart failure via accelerated resolution of inflammation in the mouse MI model ${ }^{38}$. However, the function of IRF5 in BMMSCs has not been reported elsewhere. Of the IRF family members, only IRF5 was highly induced in BMMSCs following coculture with macrophages. 


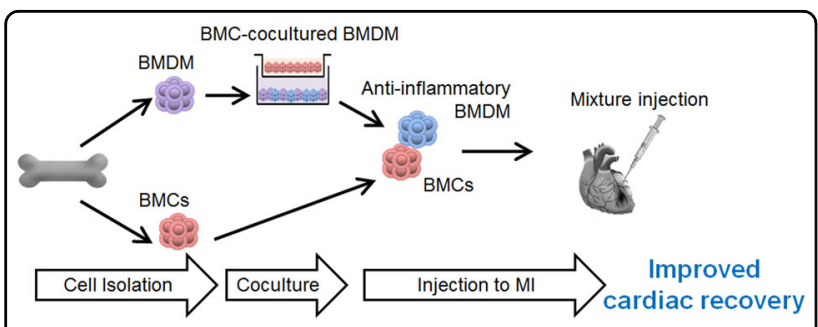

Fig. 7 Schematic representation of the contribution of BMMSCprimed BMDMs to the overall improvement in BMC-induced cardiac repair

Moreover, knockdown of IRF5 in BMMSCs blunted their anti-inflammatory activity against LPS-stimulated macrophages. The mechanism by which BMMSCs regulate inflammatory pathways is incompletely understood. IRF5 may be a supportive factor for BMMSCs in terms of immune modulatory activity.

From a clinical perspective, the most prominent advantage of using BMMSCs and BMDMs is that these two cell types originate from the same donor's bone marrow. In other words, bone marrow aspirated from a patient is sufficient to produce both BMMSCs and BMDMs for improved cell therapy. This protocol does not require additional pretreatments with reagents, genetic modifications, or differentiation induction for functional enhancement.

Our study had several limitations. First, we experienced technical difficulties with siRNA transfection in rBMMSCs. Second, we were not successful in distinguishing the injected BMDMs from resident macrophages in the infarcted myocardium.

Overall, this study showed that BMMSCs and BMDMs harvested from the same donor could be applied for cell therapy following by a coculture procedure. Cotransplantation of BMMSCs and educated BMDMs successfully induced significant functional and structural improvement in the MI rat model. Taken together, these data suggest that induction of IRF5 in BMMSCs is essential for suppressing anti-inflammatory phenotypic changes of macrophages. We suggest that BMDMs may be a feasible and effective adjuvant candidate to enhance stem cell therapy (Fig. 7).

\section{Acknowledgements}

This work was supported by the National Research Foundation of Korea (NRF2015M3A9B4066496, 2015M3A9C6031684, and 2016R1D1A1A09917796) and Chonnam National University Hospital Biomedical Research Institute (BCRI18017).

\footnotetext{
Author details

'Cell Regeneration Research Center, Chonnam National University Hospital, Gwangju 61469, Republic of Korea. ${ }^{2}$ Biomedical Research Institute, Chonnam National University Hospital, Gwangju 61469, Republic of Korea. ${ }^{3}$ Department of Cardiology, Chonnam National University Hospital, Gwangju 61469, Republic of Korea
}

Conflict of interest

The authors declare that they have no conflict of interest.

\section{Publisher's note}

Springer Nature remains neutral with regard to jurisdictional claims in published maps and institutional affiliations.

Received: 6 April 2018 Revised: 11 July 2018 Accepted: 18 July 2018. Published online: 5 November 2018

\section{References}

1. Hare, J. M. et al. Comparison of allogeneic vs autologous bone marrowderived mesenchymal stem cells delivered by transendocardial injection in patients with ischemic cardiomyopathy: the POSEIDON randomized trial. JAMA 308, 2369-2379 (2012).

2. Pittenger, M. F. et al. Multilineage potential of adult human mesenchymal stem cells. Science 284, 143-147 (1999).

3. Nauta, A. J. \& Fibbe, W. E. Immunomodulatory properties of mesenchymal stromal cells. Blood 110, 3499-3506 (2007).

4. Choudry, F. et al. A randomized double-blind control study of early intracoronary autologous bone marrow cell infusion in acute myocardial infarction: the REGENERATE-AMI clinical trialdagger. Eur. Heart J. 37, 256-263 (2016).

5. Cho, D. I. et al. The optimization of cell therapy by combinational application with apicidin-treated mesenchymal stem cells after myocardial infarction. Oncotarget 8, 44281-44294 (2017).

6. Hahn, J. Y. et al. Pre-treatment of mesenchymal stem cells with a combination of growth factors enhances gap junction formation, cytoprotective effect on cardiomyocytes, and therapeutic efficacy for myocardial infarction. J. Am. Coll. Cardiol. 51, 933-943 (2008).

7. Hu, X. et al. Transplantation of hypoxia-preconditioned mesenchymal stem cells improves infarcted heart function via enhanced survival of implanted cells and angiogenesis. J. Thorac. Cardiovasc. Surg. 135, 799-808 (2008).

8. Nahrendorf, M. et al. The healing myocardium sequentially mobilizes two monocyte subsets with divergent and complementary functions. J. Exp. Med. 204, 3037-3047 (2007).

9. Leblond, A. L. et al. Systemic and Cardiac Depletion of M2 Macrophage through CSF-1R Signaling Inhibition Alters Cardiac Function Post Myocardial Infarction. PLOS ONE 10, e0137515 (2015).

10. Ben-Mordechai, T. et al. Macrophage subpopulations are essential for infarct repair with and without stem cell therapy. J. Am. Coll. Cardiol. 62, 1890-1901 (2013).

11. Cho, D. I. et al. Mesenchymal stem cells reciprocally regulate the M1/M2 balance in mouse bone marrow-derived macrophages. Exp. Mol. Med. 46, e70 (2014).

12. Libby, P. Inflammation in atherosclerosis. Nature 420, 868-874 (2002).

13. Han, J. et al. Dual Roles of Graphene Oxide To Attenuate Inflammation and Elicit Timely Polarization of Macrophage Phenotypes for Cardiac Repair. ACS Nano 12, 1959-1977 (2018).

14. Jeong, $H$. Y. et al. 5-Azacytidine modulates interferon regulatory factor 1 in macrophages to exert a cardioprotective effect. Sci. Rep. 5, 15768 (2015).

15. Sager, H. B. et al. Proliferation and recruitment contribute to myocardial macrophage expansion in chronic heart failure. Circ. Res. 119, 853-864 (2016).

16. Heidt, $T$. et al. Differential contribution of monocytes to heart macrophages in steady-state and after myocardial infarction. Circ. Res. 115, 284-295 (2014).

17. Lee, S. J. et al. Enhanced therapeutic and long-term dynamic vascularization effects of human pluripotent stem cell-derived endothelial cells encapsulated in a nanomatrix gel. Circulation 136, 1939-1954 (2017).

18. Sano, T. et al. Impact of cardiac progenitor cells on heart failure and survival in single ventricle congenital heart disease. Circ. Res. 122, 994-1005 (2018).

19. Pandey, A. C., Lancaster, J. J., Harris, D. T., Goldman, S. \& Juneman, E. Cellular therapeutics for heart failure: focus on mesenchymal stem cells. Stem Cells Int 2017, 640108 (2017).

20. Nguyen, P. K., Rhee, J. W. \& Wu, J. C. Adult stem cell therapy and heart failure, 2000 to 2016: a systematic review. JAMA Cardiol. 1, 831-841 (2016).

21. Bolli, R. et al. Rationale and design of the CONCERT-HF trial (Combination of Mesenchymal and c-kit(+) cardiac stem cells as regenerative therapy for heart failure. Circ. Res. 122, 1703-1715 (2018). 
22. Medzhitov, R. \& Horng, T. Transcriptional control of the inflammatory response. Nat. Rev. Immunol. 9, 692-703 (2009).

23. Fraccarollo, D., Galuppo, P. \& Bauersachs, J. Novel therapeutic approaches to post-infarction remodelling. Cardiovasc. Res. 94, 293-303 (2012).

24. Harel-Adar, T. et al. Modulation of cardiac macrophages by phosphatidylserine-presenting liposomes improves infarct repair. Proc. Natl Acad. Sci. USA 108, 1827-1832 (2011).

25. Birge, R. B. et al. Phosphatidylserine is a global immunosuppressive signal in efferocytosis, infectious disease, and cancer. Cell Death Differ. 23, 962-978 (2016).

26. Frantz, S. et al. Monocytes/macrophages prevent healing defects and left ventricular thrombus formation after myocardial infarction. FASEB J. 27, 871-881 (2013).

27. van Amerongen, M. J., Harmsen, M. C., van Rooijen, N., Petersen, A. H. \& van Luyn, M. J. Macrophage depletion impairs wound healing and increases left ventricular remodeling after myocardial injury in mice. Am. J. Pathol. 170 818-829 (2007).

28. de Couto, G. et al. Macrophages mediate cardioprotective cellular postconditioning in acute myocardial infarction. J. Clin. Invest. 125, 3147-3162 (2015).

29. Nemeth, K. et al. Bone marrow stromal cells attenuate sepsis via prostaglandin $\mathrm{E}(2)$-dependent reprogramming of host macrophages to increase their interleukin-10 production. Nat. Med. 15, 42-49 (2009).
30. Powell, R. J. et al. Cellular therapy with Ixmyelocel-T to treat critical limb ischemia: the randomized, double-blind, placebo-controlled RESTORE-CLI trial. Mol. Ther. 20, 1280-1286 (2012).

31. Ledford, K. J., Zeigler, F. \& Bartel, R. L. Ixmyelocel-T, an expanded multicellular therapy, contains a unique population of M2-like macrophages. Stem Cell Res. Ther. 4, 134 (2013).

32. Ledford, K. J., Murphy, N., Zeigler, F. \& Bartel, R. L. Potential beneficial effects of ixmyelocel-T in the treatment of atherosclerotic diseases. Stem Cell Res. Ther. $\mathbf{4}$, 135 (2013).

33. Henry, T. D. et al. Safety and efficacy of ixmyelocel-T: an expanded, autologous multi-cellular therapy, in dilated cardiomyopathy. Circ. Res. 115, 730-737 (2014).

34. Patel, A. N. et al. Ixmyelocel-T for patients with ischaemic heart failure: a prospective randomised double-blind trial. Lancet 387, 2412-2421 (2016).

35. Ericson, J. A. et al. Gene expression during the generation and activation of mouse neutrophils: implication of novel functional and regulatory pathways. PLOS ONE 9, e108553 (2014).

36. Krausgruber, T. et al. IRF5 promotes inflammatory macrophage polarization and TH1-TH17 responses. Nat. Immunol. 12, 231-238 (2011).

37. Takaoka, A. et al. Integral role of IRF-5 in the gene induction programme activated by Toll-like receptors. Nature 434, 243-249 (2005).

38. Courties, $\mathrm{G}$. et al. In vivo silencing of the transcription factor IRF5 reprograms the macrophage phenotype and improves infarct healing. J. Am. Coll. Cardiol. 63, 1556-1566 (2014) 